\title{
Morphologically defined sub-stages of C. elegans vulval development in the fourth larval stage
}

\author{
Darren Z. L. Mok', Paul W. Sternberg ${ }^{2}$ and Takao Inoue ${ }^{1,2^{*}}$
}

\begin{abstract}
Background: During the fourth larval (L4) stage, vulval cells of C. elegans undergo extensive morphogenesis accompanied by changes in gene expression. This phase of vulval development, occurring after the well-studied induction of vulval cells, is not well understood but is potentially a useful context in which to study how a complex temporal sequence of events is regulated during development. However, a system for precisely describing different phases of vulval development in the $L 4$ stage has been lacking.

Results: We defined ten sub-stages of $L 4$ based on morphological criteria as observed using Nomarski microscopy (L4.0 L4.9). Precise timing of each sub-stage at $20^{\circ} \mathrm{C}$ was determined. We also re-examined the timing of expression for several gene expression markers, and correlated the sub-stages with the timing of other developmental events in the vulva and the uterus.

Conclusions: This scheme allows the developmental timing of an $L 4$ individual to be determined at approximately one-hour resolution without the need to resort to time course experiments. These well-defined developmental stages will enable more precise description of gene expression and other developmental events.
\end{abstract}

Keywords: C. elegans, Vulval development, Developmental timing, Gene expression

\section{Background}

For over 30 years, the vulval development of C. elegans has been an important model in which to study mechanisms underlying the development of complex organisms $[1,2]$. However, most studies of vulval development focused on cell fate specification and inductive interaction during the third larval (L3) stage [3]. The subsequent development of induced vulval cells, during which cell fates determined in the L3 stage are "executed" has been less studied. During the fourth larval (L4) stage, these cells undergo a complex series of morphogenetic events accompanied by dynamic changes in gene expression patterns. This makes it a potentially powerful system in which to study gene regulation during terminal differentiation and mechanisms that underlie complex morphogenetic processes [4-10].

\footnotetext{
*Correspondence: bchti@nus.edu.sg

${ }^{1}$ Department of Biochemistry, Yong Loo Lin School of Medicine, National

University of Singapore, 8 Medical Drive, Blk MD7, \#02-06, Singapore 117597, Singapore

${ }^{2} \mathrm{HHMl}$ and Division of Biology and Biological Engineering, California Institute of Technology, Pasadena, CA 91125, USA
}

Recent studies of vulval development during the L4 stage follow the detailed description published in 1999 [7]. This work, based on electron microscopy of serial sections and fluorescent labeling of cell-cell junctions, revealed the general sequence of events during the L4 stage. First, vulval cells migrate from where they were generated (near the original positions of vulval precursor cells induced in the L3 stage) toward the center of the future vulva. Second, these cells extend processes and fuse with one another such that they form a dorsal/ventral stack of seven toroids, called vulF, vulE, vulD, vulC, vulB2, vulB1 and vulA. Most of these toroids are syncytial cells with two or four nuclei. The only exceptions are vulB1 and vulB2, which remain unfused, but nevertheless arrange themselves in a ring configuration. Subsequently, the shapes of these cells change further, forming the adult structure that serves as the conduit for developing embryos and for sperm when mating with a male. During this process, additional cell-cell connections are made; vulC and vulD make connections to the vulval muscle cells that open the vulva during egg laying, vulE makes a structural connection to lateral hypodermal 
cells and vulF makes a connection with uv1 cells of the uterus.

Additional studies led to identification of a number of genes involved in this stage of vulval development and understanding of some morphogenetic processes. Polarized migration of vulval cells requires the signaling protein SMP-1/semaphorin and its receptor PLX-1/plexin, as well as small GTPases MIG-2 and CED-10 (members of the Rho/Rac family) and the GTP/GDP exchange factor UNC-73/Trio [8]. Some of these proteins show polarized localization in each vulval cell. Fusion of vulval cells into syncytial toroids requires fusogens AFF-1 and EFF-1 $[11,12]$. The zinc finger transcription factor VAB23 is a target of regulation by the EGF pathway during the L3 stage, and regulates expression of genes including smp-1, thereby linking vulval induction to regulation of morphogenesis in the L4 stage [13]. Finally, morphogenetic movements that shape the developing vulva are a result of complex interplay of various forces operating among the vulval toroids [9]. These forces include contraction of ventral toroids, requiring contraction of actin microfilaments and regulated by the Rho kinase LET502 [9], as well as generation of dorsal lumen through transient invasion of the anchor cell into the developing vulva [10].

A separate line of investigation looked at genes that are differently expressed in the seven cell types and mutations that affect their expression. Approximately 30 genes are now known to exhibit cell type specific expression among vulval cells in the L4 and/or the adult stage ([14] and references therein). Importantly, each cell type expresses a unique combination of genes, while each gene may be expressed in a single vulval cell type or in multiple cell types. Moreover, the timing of gene expression shows considerable complexity. Expression of different genes in a single cell type can initiate at different time points, and expression of a single gene in different vulval cell types can start at different time points.

The progress in understanding how expression of these genes is controlled has been slow, probably because it is relatively difficult to isolate mutations that affect cell fate or gene expression during the L4 stage. This may be because many of the genes involved in this stage of vulval development are pleiotropic and are required for earlier stages of development $[15,16]$. Among the classical lineage mutants studied by Horvitz et al., only lin-11 appears to have a phenotype consistent with cell fate change at this stage $[17,1]$. Additional genes (e.g. lin-29, egl-38, cog-1, bed-3, nhr-67, vab-23) were isolated from other screens [18-22, 13]. However, many more genes are likely to regulate this stage of vulval development given the complexity of this system.

Among the known genes regulating gene expression in the L4 stage vulva, a subset demonstrates a possible connection to the heterochronic pathway regulating stage-specific gene expression. In particular, lin-29 (encoding a zinc-finger transcription factor) is a wellknown heterochronic gene regulating the L4-to-adult transition [23]. Moreover, bed-3 (encoding a BED-type zinc-finger transcription factor) was recently discovered to be regulated by blmp-1, another component of the heterochronic pathway [24] (our results not shown). These results suggest that the timing mechanism operating throughout the entire body of the worm feeds into vulval development at specific time points, allowing for precise temporal control of gene expression. However, details of how the temporal sequence of events is regulated within the L4 stage, and how the heterochronic pathway regulates this sequence, are unclear.

In relation to these possibilities, one limitation of previous analyses of L4 development has been the lack of precise timing information. In various contexts, L4 stage animals were classified as "early", "middle" and "late" without a precise definition of each phase (e.g. $[4,5])$. In order to fully understand vulval development in the L4 stage, further studies must rely on improved description of developmental timing. Here, we present a further subdivision of the L4 stage into sub-stages (L4.0 to L4.9) based on morphological criteria in the vulva as observed by Nomarski differential interference contrast microscopy. This scheme allows staging of an L4 animal at approximately one hour resolution without the need to follow an individual animal over the course of its development. We correlate our sub-stage scheme with developmental timing when the worms are grown at $20{ }^{\circ} \mathrm{C}$. We also present improved measurement of gene expression timing for several well-characterized vulva-expressed genes.

\section{Results}

\section{Description of L4 sub-stages defined by morphological characteristics of the vulva}

To facilitate classification of L4 animals into different developmental sub-stages, we selected morphological criteria that are observed only at or after a particular point during development. The entire set of criteria selected and examples of Nomarski images are shown in Table 1 and Fig. 1. For example, in the early L4 stage, the apex of the vulval lumen lies within the vulval tissue and is pointed at the dorsal end. However, later in development, the lumen extends to the top of the vulval cell layer and the dorsal end becomes flattened.

To determine the actual timing of each sub-stage, we synchronized populations of wild-type $C$. elegans at the L3-to-L4 lethargus. These populations were then allowed to grow for specific durations at $20{ }^{\circ} \mathrm{C}$ and staged according to Table 1 (Methods, Fig. 2). At each time point, two to several stages were observed simultaneously. This 
Table 1 Sub-stages of $L 4$

\begin{tabular}{|c|c|c|c|}
\hline $\begin{array}{l}\text { Sub- } \\
\text { stage }\end{array}$ & Description & $\begin{array}{l}\text { Approximate } \\
\text { time from } \\
\text { middle of } \\
\text { the L3-to-L4 } \\
\text { lethargus }\end{array}$ & $\mathrm{n}^{*}$ \\
\hline L4.0 & $\begin{array}{l}\text { vulA, vulB and vulE have divided, } \\
\text { but vulC and vulF have not. }\end{array}$ & 0.3 & 11 \\
\hline L4.1 & $\begin{array}{l}\text { vulC and vulF have divided and } \\
\text { a narrow lumen has formed. }\end{array}$ & 1.1 & 13 \\
\hline$\llcorner 4.2$ & $\begin{array}{l}\text { Lumen has widened and a } \\
\text { prominent kink has formed } \\
\text { between vulC and vulD. }\end{array}$ & 2.8 & 31 \\
\hline$\llcorner 4.3$ & $\begin{array}{l}\text { vulFs have separated and the } \\
\text { apex of the lumen is flat and } \\
\text { capped by the anchor cell. }\end{array}$ & 4.1 & 21 \\
\hline$\llcorner 4.4$ & $\begin{array}{l}\text { utse is visible as a thin layer } \\
\text { separating the vulval and uterine } \\
\text { lumens. "Fingers" are formed at the } \\
\text { sides of the vulva next to vulB1 and vulB2. }\end{array}$ & 5.0 & 26 \\
\hline$\llcorner 4.5$ & $\begin{array}{l}\text { Side of the vulval lumen between } \\
\text { vulC and vulD forms a smooth curve. }\end{array}$ & 6.4 & 45 \\
\hline L4.6 & $\begin{array}{l}\text { "Fingers" between vulB2 and vulC are } \\
\text { pointed ventrally. }\end{array}$ & 7.5 & 23 \\
\hline$\llcorner 4.7$ & $\begin{array}{l}\text { vulFs have migrated closer such that the } \\
\text { lumen is narrowed in the dorsal section. } \\
\text { The approximate cutoff is when the width } \\
\text { of the channel is less than the width of } \\
\text { the vulD nucleus. }\end{array}$ & 8.3 & 9 \\
\hline$\llcorner 4.8$ & Lumen is partially collapsed. & 8.5 & 4 \\
\hline$\llcorner 4.9$ & Lumen is completely collapsed. & 8.8 & 5 \\
\hline Adult & & 9.7 & 9 \\
\hline
\end{tabular}

*Number of animals scored at each stage

probably reflects the fact that the L3-to-L4 lethargus is about two hours long at $20{ }^{\circ} \mathrm{C}[25,26]$. To determine the approximate timing of each sub-stage, we took the average time point for all observations of each L4 substage (Table 1, Fig. 3). We expect that this would correspond to the approximate time taken from the middle of the L3-to-L4 lethargus to the middle of a given substage. We found that most of the sub-stages were spaced apart by approximately one to two hours.

\section{Precise timing of reporter gene expression in the L4 stage}

The expression pattern of a number of genes in the L4 stage vulva has been reported (e.g. [4]). However, most of these reported expression patterns lack precise timing information. We therefore re-examined expression of ten reporters (Table 2, Fig. 4, Methods). Our results were largely consistent with approximate timing reported previously. "Early-L4" roughly corresponds to sub-stages L4.0 to L4.3, "mid-L4" roughly corresponds to sub-stages L4.4 to L4.6 and "late-L4" roughly corresponds to sub-stages L4.7 to L4.9. Some researchers have used the term "Christmas tree stage" to identify
mid-L4 animals with specific morphological features (e.g. [27]). In our scheme, this corresponds to sub-stages L4.4, L4.5 and L4.6.

Interestingly, expression of $c d h-3:: c f p$, reported to begin in early-L4, and expression of ceh-2::gfp, reported to begin in mid-L4 [4], actually started at the same substage L4.1. Although differences in assay conditions could account for the discrepancy, this is perhaps more likely due to differences in the definition of "early-L4" and "mid-L4" used in different experiments, highlighting the need for better staging of L4 animals.

\section{Correlation of L4 sub-stages with other developmental events}

We also correlated L4 sub-stages with timing of other developmental events in the L4 stage (Table 3, Fig. 3). Following the terminal division, vulval cells undergo migration and fusion [7]. Using a strain carrying ajm$1:: g f p$, an adherens junction specific GFP reporter, we examined the state of cell migration and fusion in L4 sub-stages (Methods, Fig. 5). We found that cell migration continues from L3 into sub-stage L4.1. By the L4.2 sub-stage, in most animals examined (4 of 5), all cells had connected with their counterparts from the opposite side of the vulva. As for cell fusion, an animal examined in the L4.1 sub-stage had not fused its anterior and posterior cells $(n=1)$. However, in the L4.2 substage, anterior/posterior fusion of vulA, vulC and vulD was observed in some animals ( 4 of 5 ), and by the L4.3 sub-stage, vulA, vulC and vulD were fused in all animals $(n=6)$. Therefore, cell migration is complete by the L4.2 sub-stage and cell fusions of vulA, vulC and vulD take place within one or two hours of cells becoming attached. The next cell to fuse, vulF, was unfused in the L4.2 sub-stage $(n=4)$, sometimes fused in the L4.3 sub-stage (3 of 6) and always fused in the L4.4 sub-stage $(n=6)$. (One of four L4.5 animals examined had unfused vulF cells, suggesting there may be some individual variability in the timing of cell fusion.) Finally, vulE fusion was not observed in L4.7 animals $(\mathrm{n}=2)$, suggesting that this fusion takes place in L4.8 or later.

As with vulval development, the uterus undergoes a complex morphogenetic process during the L4 stage [28]. We did not attempt to correlate all known developmental events in the uterus with our sub-stages. Instead, we looked at an easily assayed aspect of uterine development, the generation of the uterine lumen. We found that L4.2 animals had no visible uterine lumen, whereas L4.4 animals had a large uterine lumen that appeared to be fully distended. Some animals in the L4.3 stage had an intermediate sized lumen, and it appears that the uterine lumen is generated and enlarges during this sub-stage. 

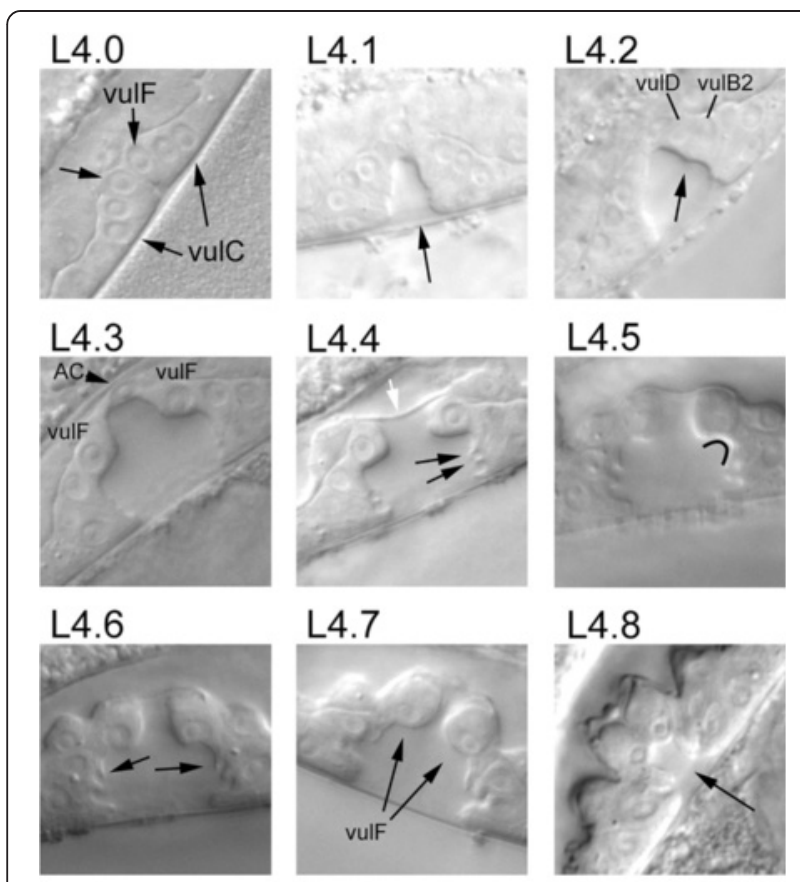

\section{L4.9}

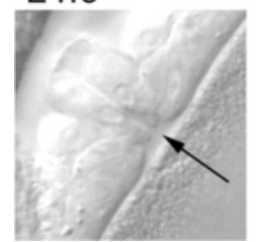

Fig. 1 Nomarski images of C. elegans vulva at different L4 sub-stages ( L4.0 - L4.9). Each sub-stage is distinguished from another by the morphological appearance of the vulva (Table 1). All images in this figure were taken in the focal plane corresponding to the midline of the worm. L4.0: arrows point to undivided cells. L4.1: arrow points to the vulval lumen. L4.2: arrow points to the "kink" between vulC and vulD cells. vulCs are out of the plane of focus. L4.3: the anchor cell and vulF cells are labeled. L4.4: the utse cell separating the vulva lumen from the uterine lumen is indicated by the white arrow. Black arrows point to "fingers" which form on sides of the vulva. L4.5: the concave curve between vulD and vulB2 is shown (black arc). L4.6: ventrally oriented "fingers" are indicated by black arrows. L4.7: the opening between vulF cells are starting to narrow. L4.8, L4.9: the vulval lumen (black arrow) collapses during these stages

\section{Discussion}

Utility of morphology-based staging in studies of late vulval development

An alternative to morphology-based staging is to carry out timed experiments. Individuals or populations at a particular developmental time (e.g. exit of the L3-to-L4 molt lethargus) can be selected, and further observations can be carried out at specific subsequent time points. The major advantage of morphology-based staging is that it is much less laborious. Using this system, any given animal in the L4 stage, provided that it has normal

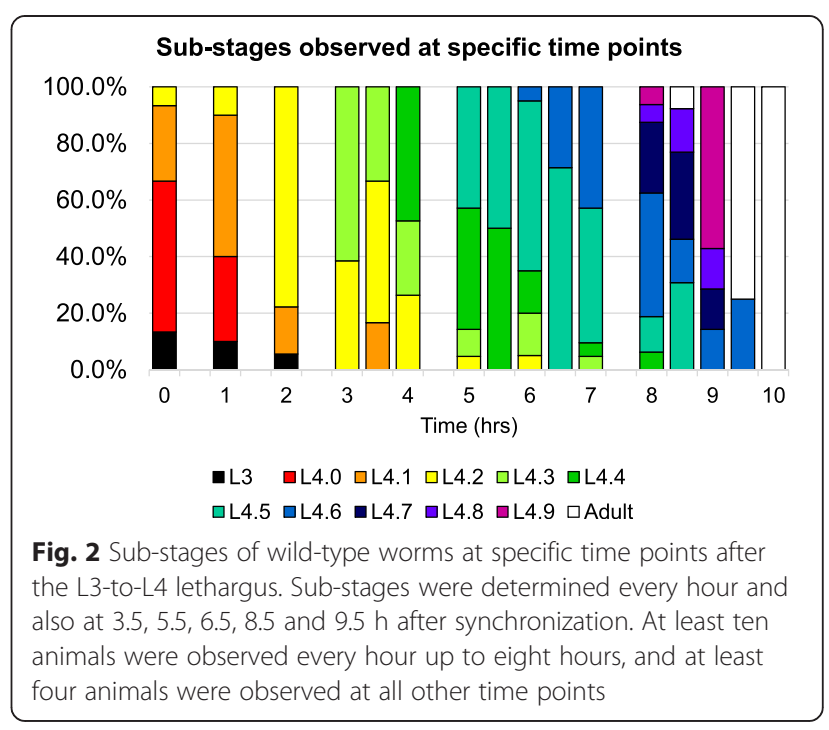

vulval development, can be staged with approximately one hour resolution.

The precise control of the temporal sequence of events during development is a problem that is not fully understood. Although the heterochronic pathway is well characterized, the sequence of events within each stage has received less attention. In particular, development of organs like the vulva requires a complex sequence of morphological changes within a single developmental stage, suggesting that there are additional timing cues which regulate specific developmental events at specific substages. Whether these timing cues are organ-specific is unknown, as is the relationship between these sub-stage cues and the heterochronic pathway. L4 sub-stages described in this report should facilitate the analysis of morphogenetic or gene expression events which occur at specific time points within the L4 stage.

\section{Conclusions}

Communication and reproducibility of results is enhanced by definitions of terms. WormBase has developed an ontology of nematode life stages that reconciles most of the terms used in the literature (W. Chen and P.W.S, unpublished results). The sub-stages we have defined here have been added to the Life Stage Ontology. This addition will allow researchers to annotate gene expression and other experiments with these stages, thus providing a more accurate depiction of their observations.

\section{Methods}

\section{C. elegans culture and timing of L4 sub-stages}

C. elegans strains were cultured using standard methods on NGM agar plates seeded with OP50 at $20{ }^{\circ} \mathrm{C}$ [29]. The N2 Bristol strain was used as the wild-type. To determine the precise timing of each developmental sub- 


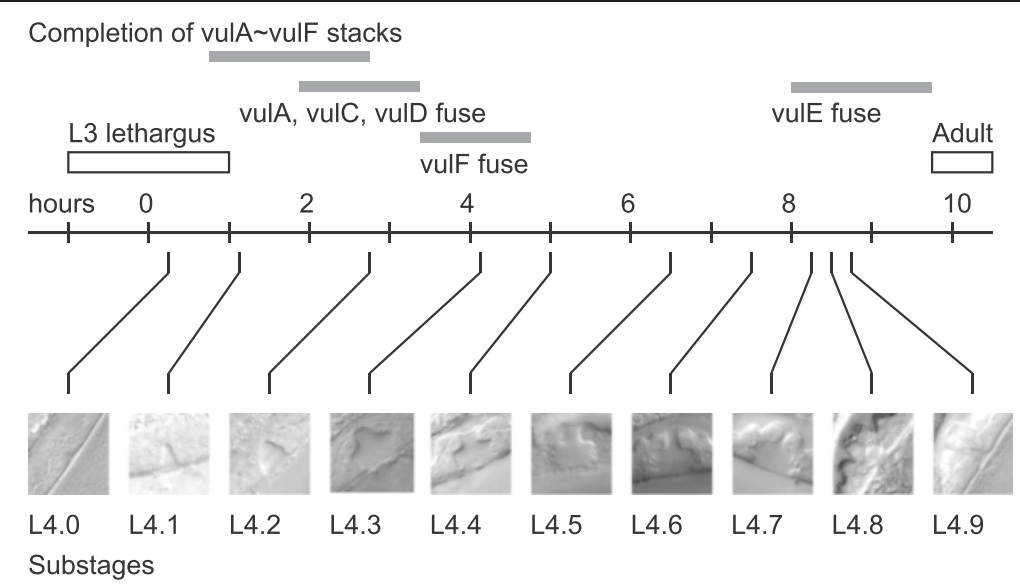

Fig. 3 Timeline of sub-stages and developmental events in the L4 stage

stage, a population of N2 wild type worms were synchronized using a modified version of the egg-laying protocol described by Lionaki and Tavernarakis (2013) [30]. First, gravid hermaphrodites were allowed to lay eggs on seeded NGM plates for one hour and were subsequently removed. Next, the plates were incubated at $20{ }^{\circ} \mathrm{C}$ for approximately $40 \mathrm{~h}$ and screened for molting L3 animals based on pale appearance, lack of motility

Table 2 Duration of expression for vulval gene expression markers

\begin{tabular}{|c|c|c|c|}
\hline Reporter & Cells & $\begin{array}{l}\text { Duration of } \\
\text { GFP } \\
\text { expression }\end{array}$ & Comments \\
\hline egl-17::gfp & C, D & L4.2 to L4.9* & [34] \\
\hline$c d h-3:: c f p$ & $\begin{array}{l}\text { C, D, } \\
E, F\end{array}$ & L4.1 to L4.9* & $\begin{array}{l}\text { [35] Variable expression in L4.1; } \\
\text { consistent in later sub-stages }\end{array}$ \\
\hline ceh-2::.gfp & $\begin{array}{l}\mathrm{B} 1, \mathrm{~B} 2, \\
\mathrm{C}\end{array}$ & L4.1 to L4.9* & $\begin{array}{l}\text { [4] Variable expression in all sub- } \\
\text { stages; low level of expression in } \\
\text { B2, C from L4.1 to L4.7 }\end{array}$ \\
\hline daf-6::cfp & $E, F$ & L4.3 to L4.9* & $\begin{array}{l}\text { [36] Expression in E variable in } L 4.3 \\
\text { and } L 4.4 \text {, consistent in later sub- } \\
\text { stages; expression in F variable } \\
\text { throughout }\end{array}$ \\
\hline B0034.1::gfp & $E, F$ & $\begin{array}{l}\text { No } \\
\text { expression in } \\
\text { L4 }\end{array}$ & [4] Expressed in adults only \\
\hline F47B8.6::gfp & $\begin{array}{l}\text { C, D, } \\
E, F\end{array}$ & $\begin{array}{l}\text { No } \\
\text { expression in } \\
\text { L4 }\end{array}$ & [4] Expressed in adults only \\
\hline \multirow[t]{2}{*}{$e g l-26:: g f p$} & $\mathrm{~B} 1, \mathrm{~B} 2$ & L4.4 to L4.9* & [32] \\
\hline & $E$ & L4.2 to L4.9* & \\
\hline egl-38::gfp & $D, E, F$ & $\llcorner 4.1$ to $L 4.7$ & $\begin{array}{l}\text { [22] Only reporter in this study } \\
\text { where expression was obviously } \\
\text { turned off during L4. }\end{array}$ \\
\hline best-13::gfp & $\begin{array}{l}B 1, B 2, \\
C, D, E\end{array}$ & L4.1 to L4.9* & $\begin{array}{l}\text { [37] Variable expression possibly } \\
\text { due to loss of extrachromosomal } \\
\text { array }\end{array}$ \\
\hline
\end{tabular}

*Expression of these reporters persist into the adult stage and absence of pharyngeal pumping. The molt-stage worms were then transferred to new seeded NGM plates. Finally, these synchronized populations were examined by Nomarski microscopy as described [25] at specific time points and the sub-stages were determined.

\section{GFP expression}

To analyze the gene expression pattern in various L4 sub-stages, semi-synchronized populations of GFP reporter strains were generated by synchronized egg-laying as described above. After 40 to $50 \mathrm{~h}$ at $20^{\circ} \mathrm{C}, \mathrm{L} 4$ animals were picked randomly and analyzed for fluorescence and the sub-stage.

Strains used are: NH2466 ayIs4[egl-17::gfp]; PS3504 syIs54[ceh-2::gfp]; PS3475 syIs51[cdh-3::gfp]; JU486 mfIs4[egl-17::yfp; daf-6::cfp]; PS3664 syIs65[B0034.1::pes-1 0::gfp]; PS3527 syIs61[F48B8.6::gfp]; MH1564 kuIs36[egl26::gfp]; OP171 wgIs171[egl-38::TY1::EGFP::3xFLAG]; BC 15833 sEx15833[best-13:::gfp]; ZF1638 qwEx197[pax-2::y fp]; ZF1639 qwEx198[pax-2::yfp] [4, 31-33].

Of the transgenic strains we examined, two (PS3664 syIs65[B0034.1::pes-10::gfp] and PS3527 syIs61[F48B8.6::g $f p]$ ) were found to be expressed only in the adult and not in L4, as reported previously [4]. The pax-2::yfp transgenes were generated in this study by fusing $3.1 \mathrm{~kb}$ sequence upstream of the $p a x-2$ gene to the $y f p$ (yellow fluorescent protein) coding region. Although this fragment is known to contain an enhancer element active in vulval cells, our reporter failed to express YFP in the vulval tissue for unknown reasons.

To assay for cell fusion, we crossed NW1615 plx1(ev724) jcIs1[ajm-1::gfp]; him-5(e1490) or NW1072 smp-1(ev715); jcIs1[ajm-1::gfp]; him-5(e1490) hermaphrodites with EG7959 unc-119(ed3); him-5(e1490) oxTi405 [eft-3p::TdTomato::H2B::unc-54 3'UTR + Cbrunc-119(+)] males and F1 progeny carrying oxTi405 were examined. Confocal images were obtained using Zeiss 


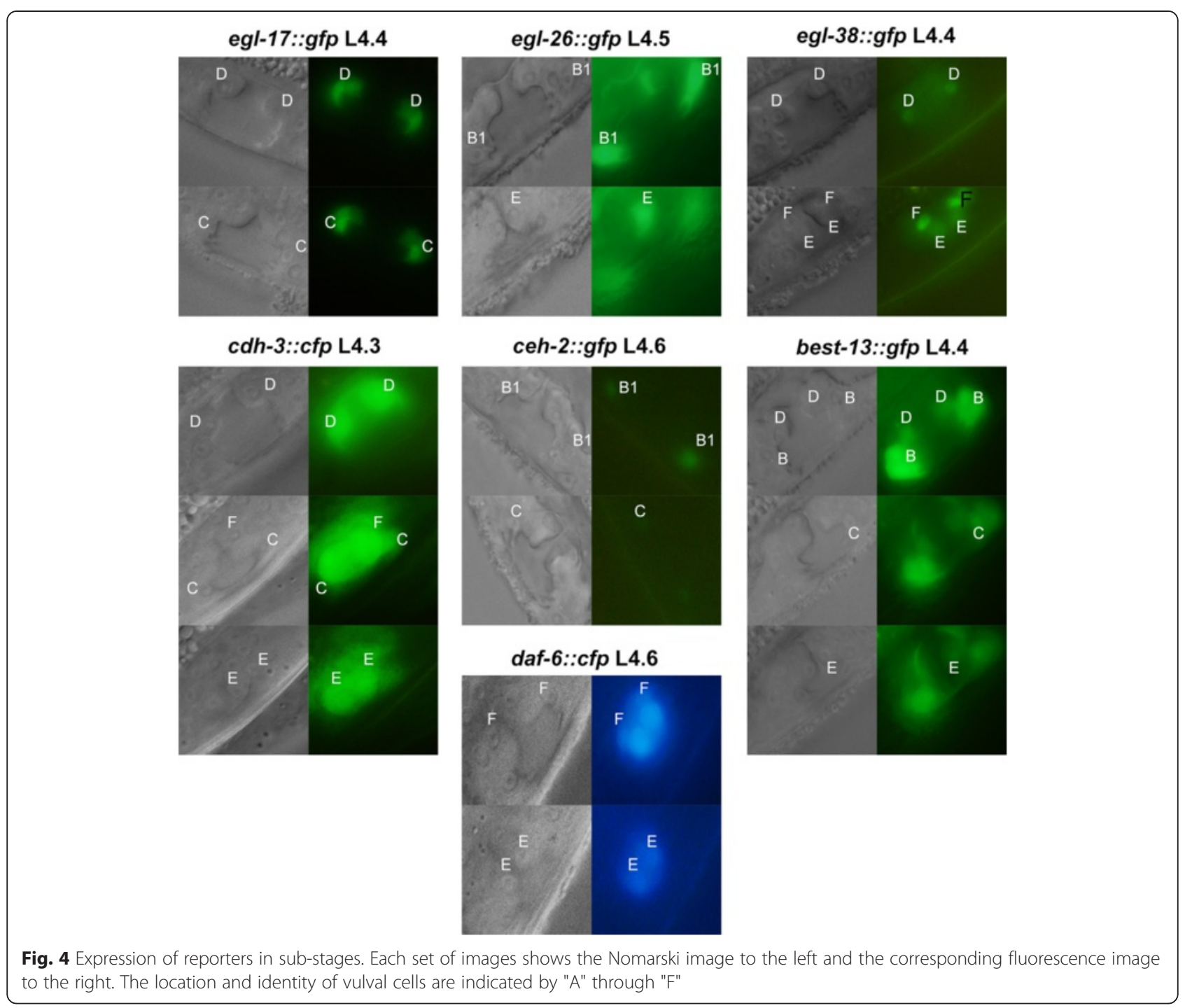

Table 3 Correlation of sub-stages with other developmental events in the L4

\begin{tabular}{|c|c|c|c|c|}
\hline \multirow[b]{2}{*}{ Stage } & \multicolumn{4}{|l|}{ Timing } \\
\hline & From L3-to-L4 molt & From hatch & Cell migration and fusion & Uterine lumen \\
\hline$\overline{L 4.0}$ & 0.3 & 34.3 & & \\
\hline$\llcorner 4.1$ & 1.1 & 35.1 & & \\
\hline$\llcorner 4.2$ & 2.8 & 36.8 & $\begin{array}{l}\text { All cells complete migration. } \\
\text { vulA, vulC and vulD cells fuse. }\end{array}$ & Uterine lumen has not started to form. \\
\hline$\llcorner 4.3$ & 4.1 & 38.1 & vulF cells fuse. & Uterine lumen starts to form. \\
\hline$\llcorner 4.4$ & 5.0 & 39.0 & & Uterine lumen is fully distended. \\
\hline$\llcorner 4.5$ & 6.4 & 40.4 & & \\
\hline$\llcorner 4.6$ & 7.5 & 41.5 & & \\
\hline$\llcorner 4.7$ & 8.3 & 42.3 & & \\
\hline$\llcorner 4.8$ & 8.5 & 42.5 & vulE cells fuse at $L 4.8$ or later & \\
\hline$\llcorner 4.9$ & 8.8 & 42.8 & & \\
\hline adult & 9.7 & 43.7 & & \\
\hline
\end{tabular}

Time from hatching is inferred based on the assumption that L3-to-L4 molt takes place around $34 \mathrm{~h}$ after hatching 


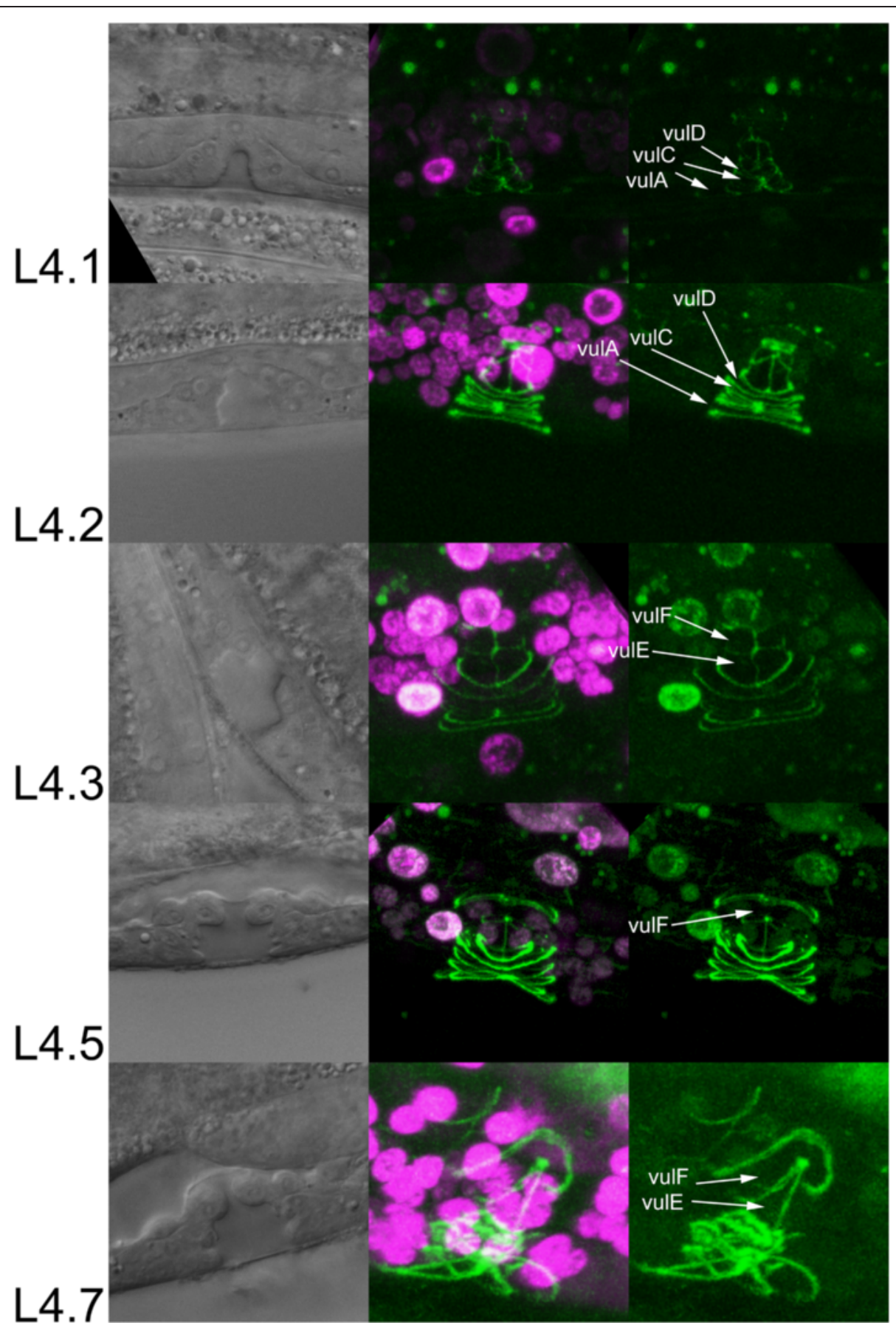

Fig. 5 Cell fusion and sub-stages. Cell-cell junctions are labeled by ajm-1::.gfp (green) whereas nuclei are labeled with TdTomato::H2S (magenta). For each stage, Nomarski (left) and confocal (right) images are from the same worm, but taken separately on different microscopes. Junctions between syncytial hypodermis, vulval cells and the uterus are visible as rings, here viewed from the side. Prior to fusion of anterior and posterior vulval cells, the boundary between these cells are visible as vertical (dorsal/ventral) line of ajm-1::gfp fluorescence. In the L4.1 animal shown, vulC and vulD cells have formed toroids but have not fused. Anterior and posterior vulA cells are still migrating but have not contacted each other. In the L4.2 animal, vulA, vulC and vulD cells have fused, indicated by the absence of ajm-1::gfp fluorescence between anterior and posterior cells. In L4.1, L4.2 and L4.3 animals shown, vulE and vulF cells have not fused. However, in the L4.5 animal shown, the boundary between anterior and posterior vulF cells is not detectable, indicating cell fusion had taken place. In the L4.7 stage, further changes to the vulval cell shapes have occurred, but vulE cells have not fused yet 
LSM700 confocal microscope. Both confocal and conventional fluorescence microscopy were used to determine whether the cells were fused.

\section{Competing interests}

The authors declare that they have no competing interests.

\section{Authors' contributions}

$\mathrm{TI}$ and PWS developed the L4 sub-staging scheme. DZLM determined the timing of sub-stages and the expression of reporters in sub-stages. TI and DZLM determined the correlation with other developmental events. The manuscript was drafted by DZLM and edited by all authors. All authors read and approved the final manuscript.

\section{Acknowledgments}

We thank Goh Kah Yee for preliminary analysis of best-13::gfp expression. We thank Marie-Anne Felix for the daf-6::cfp plasmid and strain. Some strains used in this study were provided by the Caenorhabditis Genetics Center, which is funded by NIH Office of Research Infrastructure Programs (P40 OD010440). This work was supported by the Singapore Ministry of Education Academic Research Fund Tier 1 grant (T13-1001-P02). PWS is an Investigator of the Howard Hughes Medical Institute.

Received: 18 February 2015 Accepted: 3 June 2015

Published online: 12 June 2015

\section{References}

1. Horvitz HR, Sulston JE. Isolation and genetic characterization of cell-lineage mutants of the nematode Caenorhabditis elegans. Genetics. 1980;96(2):435-54.

2. Sternberg PW. Vulval development. WormBook. 2005:1-28. doi:10.1895/ wormbook.1.6.1.

3. Sternberg PW, Horvitz HR. Pattern formation during vulval development in C. elegans. Cell. 1986;44(5):761-72.

4. Inoue T, Sherwood DR, Aspock G, Butler JA, Gupta BP, Kirouac M, et al. Gene expression markers for Caenorhabditis elegans vulval cells. Mech Dev. 2002;119(1):S203-9.

5. Inoue T, Wang M, Ririe TO, Fernandes JS, Sternberg PW. Transcriptional network underlying Caenorhabditis elegans vulval development. Proc Natl Acad Sci U S A. 2005;102(14):4972-7. doi:10.1073/pnas.0408122102.

6. Ririe TO, Fernandes JS, Sternberg PW. The Caenorhabditis elegans vulva: a post-embryonic gene regulatory network controlling organogenesis. Proc Natl Acad Sci U S A. 2008;105(51):20095-9. doi:10.1073/pnas.0806377105.

7. Sharma-Kishore R, White JG, Southgate E, Podbilewicz B. Formation of the vulva in Caenorhabditis elegans: a paradigm for organogenesis. Development. 1999;126(4):691-9.

8. Dalpe G, Brown L, Culotti JG. Vulva morphogenesis involves attraction of plexin 1-expressing primordial vulva cells to semaphorin 1a sequentially expressed at the vulva midline. Development. 2005;132(6):1387-400. doi:10.1242/dev.01694

9. Farooqui S, Pellegrino MW, Rimann I, Morf MK, Muller L, Frohli E, et al. Coordinated lumen contraction and expansion during vulval tube morphogenesis in Caenorhabditis elegans. Dev Cell. 2012;23(3):494-506. doi:10.1016/j.devcel.2012.06.019.

10. Estes KA, Hanna-Rose W. The anchor cell initiates dorsal lumen formation during C. elegans vulval tubulogenesis. Dev Biol. 2009:328(2):297-304. doi:10.1016/j.ydbio.2009.01.034.

11. Mohler WA, Shemer G, del Campo JJ, Valansi C, Opoku-Serebuoh E, Scranton $\mathrm{V}$, et al. The type I membrane protein EFF-1 is essential for developmental cell fusion. Dev Cell. 2002;2(3):355-62

12. Sapir A, Choi J, Leikina E, Avinoam O, Valansi C, Chernomordik LV, et al. AFF1, a FOS-1-regulated fusogen, mediates fusion of the anchor cell in C. elegans. Dev Cell. 2007:12(5):683-98. doi:10.1016/j.devcel.2007.03.003.

13. Pellegrino MW, Farooqui S, Frohli E, Rehrauer H, Kaeser-Pebernard S, Muller F, et al. LIN-39 and the EGFR/RAS/MAPK pathway regulate C. elegans vulval morphogenesis via the VAB-23 zinc finger protein. Development. 2011:138(21):4649-60. doi:10.1242/dev.071951.

14. Gupta BP, Hanna-Rose W, Sternberg PW. Morphogenesis of the vulva and the vulval-uterine connection. WormBook. 2012:1-20. doi:10.1895/ wormbook.1.152.1.
15. Chamberlin HM, Palmer RE, Newman AP, Sternberg PW, Baillie DL, Thomas $\mathrm{JH}$. The PAX gene egl-38 mediates developmental patterning in Caenorhabditis elegans. Development. 1997;124(20):3919-28.

16. Gissendanner CR, Crossgrove K, Kraus KA, Maina CV, Sluder AE. Expression and function of conserved nuclear receptor genes in Caenorhabditis elegans. Dev Biol. 2004:266(2):399-416.

17. Ferguson EL, Sternberg PW, Horvitz HR. A genetic pathway for the specification of the vulval cell lineages of Caenorhabditis elegans. Nature. 1987;326(6110):259-67. doi:10.1038/326259a0.

18. Newman AP, Inoue T, Wang M, Sternberg PW. The Caenorhabditis elegans heterochronic gene lin-29 coordinates the vulval-uterine-epidermal connections. Curr Biol. 2000;10(23):1479-88.

19. Palmer RE, Inoue T, Sherwood DR, Jiang LI, Sternberg PW. Caenorhabditis elegans cog-1 locus encodes GTX/NkX6.1 homeodomain proteins and regulates multiple aspects of reproductive system development. Dev Biol. 2002;252(2):202-13

20. Fernandes JS, Sternberg PW. The tailless ortholog $n$ hr-67 regulates patterning of gene expression and morphogenesis in the C. elegans vulva. PLoS Genet. 2007;3(4):e69. doi:10.1371/journal.pgen.0030069.

21. Inoue T, Sternberg PW. C. elegans BED domain transcription factor BED-3 controls lineage-specific cell proliferation during organogenesis. Dev Biol. 2010;338(2):226-36. doi:10.1016/j.ydbio.2009.12.005.

22. Rajakumar V, Chamberlin HM. The Pax2/5/8 gene eg/-38 coordinates organogenesis of the C. elegans egg-laying system. Dev Biol. 2007;301(1):240-53. doi:10.1016/j.ydbio.2006.08.068

23. Rougvie $A E$, Ambros $V$. The heterochronic gene lin-29 encodes a zinc finger protein that controls a terminal differentiation event in Caenorhabditis elegans. Development. 1995:121(8):2491-500.

24. Horn M, Geisen C, Cermak L, Becker B, Nakamura S, Klein C, et al. DRE-1/ FBXO11-dependent degradation of BLMP-1/BLIMP-1 governs C. elegans developmental timing and maturation. Dev Cell. 2014;28(6):697-710. doi:10.1016/j.devcel.2014.01.028.

25. Sulston JE, Horvitz HR. Post-embryonic cell lineages of the nematode, Caenorhabditis elegans. Dev Biol. 1977;56(1):110-56.

26. Van Buskirk C, Sternberg PW. Epidermal growth factor signaling induces behavioral quiescence in Caenorhabditis elegans. Nat Neurosci. 2007:10(10):1300-7. doi:10.1038/nn1981.

27. Eisenmann DM, Kim SK. Protruding vulva mutants identify novel loci and Wnt signaling factors that function during Caenorhabditis elegans vulva development. Genetics. 2000;156(3):1097-116.

28. Newman AP, White JG, Sternberg PW. Morphogenesis of the C. elegans hermaphrodite uterus. Development. 1996;122(11):3617-26.

29. Brenner S. The genetics of Caenorhabditis elegans. Genetics. 1974;77(1):7194.

30. Lionaki E, Tavernarakis N. High-throughput and longitudinal analysis of aging and senescent decline in Caenorhabditis elegans. Methods Mol Biol. 2013;965:485-500. doi:10.1007/978-1-62703-239-1_32.

31. Hunt-Newbury R, Viveiros R, Johnsen R, Mah A, Anastas D, Fang L, et al. High-throughput in vivo analysis of gene expression in Caenorhabditis elegans. Plos Biol. 2007;5(9):1981-97. doi:10.1371/journal.pbio.0050237.

32. Hanna-Rose W, Han M. The Caenorhabditis elegans EGL-26 protein mediates vulval cell morphogenesis. Dev Biol. 2002;241(2):247-58. doi:10.1006/ dbio.2001.0514.

33. Niu W, Lu ZJ, Zhong M, Sarov M, Murray Jl, Brdlik CM et al. Diverse transcription factor binding features revealed by genome-wide ChIP-seq in C. elegans. Genome Res. 2011. doi:10.1101/gr.114587.110

34. Burdine RD, Branda CS, Stern MJ. EGL-17(FGF) expression coordinates the at traction of the migrating sex myoblasts with vulval induction in C. elegans. Development. 1998;125(6):1083-93.

35. Pettitt J, Wood WB, Plasterk RH. cdh-3, a gene encoding a member of the cadherin superfamily, functions in epithelial cell morphogenesis in Caenorhabditis elegans. Development. 1996;122(12):4149-57.

36. Perens EA, Shaham S. C. elegans daf-6 encodes a patched-related protein required for lumen formation. Dev Cell. 2005;8(6):893-906. doi:10.1016/ j.devcel.2005.03.009.

37. McKay SJ, Johnsen R, Khattra J, Asano J, Baillie DL, Chan S, et al. Gene expression profiling of cells, tissues, and developmental stages of the nematode C elegans. Cold Spring Harb Symp Quant Biol. 2003:68:159-69. 